\title{
Integrated Predicative Synchronization Implemented in a Federated DBMS*
}

\author{
Stefan Böttcher \\ IBM Deutschland GmbH \\ Scientific Center \\ Institute for Knowledge Based Systems \\ Postfach 800880 \\ D-7000 Stuttgart 80 \\ West Germany \\ e-mail: BOETTCHE@DSOLILOG.bitnet
}

\section{Abstract}

An important implementation aspect of federated database systems is the implementation of the transaction synchronization component. Most approaches in database transaction synchronization are based on a single synchronization strategy, e.g. locking or validation. However, the adequacy of a transaction synchronization strategy often depends on the expected probability of conflicts. Therefore, it seems to be ndvantageous to adapt the synchronization strategy to the expected conflict probability. This paper describes an integrated scheduler based on predicative locking and predicative validation, which adapts its synchronization strategy to the expected conflict probability.

A comparison of predicative locking and predicative validation shows that predicative locking is superior to predicative validation if many conflicts occur, whereas predicative validation performs better if no conflicts occur. In order to combine the advantages of both synchronization strategies and to avoid their disadvantages, we develop an integration of both strategies. The integrated synchronization strategy uses an efficient heuristics in order to choose the appropriate synchronization strategy. An implementation of all three predicative synchronization strategies (locking, validation and their adaptive integration) within a single federated database system shows that the integrated synchronization is superior to both predicative locking and predicative validation.

"This work has been partially done ot the University of Frankfurt and has been supported by the Deutsche Forschungsgemeinschaft under Grant-No SCHM350/3-1.

\section{Introduction}

A federated database system consists of a server contrining the database and a couple of workstations which concurrently execute transactions of application programs. The transactions are synchronized by a scheduler the main part of which is located on the server and schedules the access to the database. We argue that the appropriate synchronization strategy for accesses to this database may be very much npplication dependent and therefore the server should integrate more than one synchronization strategy.

This paper presents an integrated predicative synclironization strategy based on both predicative locking and predicative validation. This integrated synchronization uses an efficient heuristics in order to choose an appropriate synchronization strategy. The henristics is motivated by a comparison of predicative locking and predicative validation. It is further confirmed by performance tests for a given set of transactions. The performance tests compare predicative locking, predicative validation, and the presented integration of both using an implementation of these strategies within the DBPL database system [Böttcher, 1989]. Previous perCorunnce evaluations in the DBPL databnse system have shown that the time needed for predicative locking is less tham $0.1 \%$ of the trnusaction run time on the databnse server, if predicative locking nses an incomplete theorem prover [Böttcher et al., 1986]. Therefore predicative syuchironization is cousidered to be not too expensive.

Correctness of transaction scheduling and a high performance are considered to be the most important propertics of a syuchronization strategy for 
transactions accessing a relational database (e.g. [Papadimitrion, 1986]). Correctness of transaction scheduling and especially the phantom problem [Eswaran et al., 1976], [Bernstein et al., 1981], require to use predicative synchrouization instead of physical synchronisation. On the other hand, performance comparisons have only been given for plysical synchronization (e.g. [Franaszek and Robinson, 1985]) but not for predicative synchronization.

Performance results comparing predicative locking and predicative validation can not be expected to be the same as for a comparison of physical locking and physical validation, because physical locking uses a simple locking mechanism whereas predicative locking uses a fast theorem prover (e.g. [Böttcher et al., 1986]) in order to check lock compatibility. Sinvilarly, performance results comparing predicative locking and predicative validation with an integration of both may differ from a comparison of physical locking and physical validation with an integration of both of these strategies.

That is why this paper describes an integrated predicative synchronization strategy, and furthermore summarizes the result of a performance test for a given set of transactions, which compares an implementation of predicative locking, predicative validation and the pre-
sented integration of both.

The presented integration of locking and validation can not only be applied to queries and write operations on relational databases, but also to object-oriented and to the using attribute inheritance [Böttcher, 1990a] write operation

The paper is organized as follows. The next section summarizes the differences between predicative symchronization and physical synchronization. The third section discusses the advantages of both synchronization strategy. The fourents an integrated synchronizaplementation of the integrated characterizes the imegy and outlines the resilted synchronization stratison.

\section{Predicative synchronization}

The phantom problem is solved by both predicative locking and predicative validation, but not by physical [Reimer, 1983].

\subsection{The difference between predicative locking and physical locking}

Detailed descriptions of predicate locking are given e.g. in [Eswaran et al., 1976], [Bernstein el al., 1981] The difference of predicate locking compared to phys ical locking is that transactions lock predicatively de scribed subrelations instead of tuples or physical rela tions. Subrelations are subsets of the relation schems of the corresponding relation. Two locks on snbrela tions of the snme relation are compatible, if the subrelations locked do not ove rlap, i.e. if there does not exist any tuple in the corresponding relation schema which is contained in both subrelations. This overlapping of subrelations is clecked by $n$ fast theorem prover.

In order to get a fast theorem prover for predicative locking, we nllow that it is incomplete, i.e. the theorem prover may decide, that it can not find out whether or not two subrelations overlap. In this case (and if the subrelations overlap), the scheduler delays one of the transactions. Only if the theorem prover finds out that both transactions can run in parallel, the scheduler allows them to run in parallel. As described in [Böttcher et al., 1.986] the theorem prover has a contplexity of $O\left(n^{3}\right)$ and finds ont all typical cases where both transactions can run in parallel. ${ }^{1}$

The time needed for predicative locking could be reduced to less than $0.1 \%$ of the transaction run tinc, because the DBPL databnse uses an incomplete the orem prover. Therefore predicative synchronization is considered to be not too expensive.

\subsection{The difference between predicative validation and physical validation}

Predicative validation is described in [Reimer, 1983]. The division of transactions into phases, the assignment of transaction numbers, nud the rule which transaction has to validate agninst which other transactions are equal to the parallel validation algorithm given for pliysical validation [Kung and Robinson, 1981]. The difference between predicative validation and physical validation lies in the evaluntion of the validation condition.

As in physical validation, every transaction allocates " rend set and a write for every relation it accesses. In

iThe

in cases, where the theorem prover can not find out in a time $O\left(n^{3}\right)$ whether or not two subrelations overlap, are only of theoretical interest, but usually do not ocenr in practice. 
predicative validation, the read set of a database relation contains the subrelations accessed by queries of the transaction ${ }^{2}$, and the write set contains old and new values of tuples written by the transaction. The validation condition of predicative validation is the following: Two operations of two trausactions are in conflict, if there is a subrelation contained in the read set of one transaction and there is a tuple in a write set of the other transaction so that the tuple is contained in the subrelation. This containment is checked by the query evaluation system.

Because of these differences between predicative validation and physical validation and between predicative locking and physical locking a comparison of predicative validation and predicative locking may lead to different results from a comparison of physical validation and physical locking.

\section{Comparing and integrating predicative validation and predicative locking}

On the basis of a discussion of the advantages and disadvantages of predicative validation and predicative locking, we present an integration of both strategies with the aim to combine the advantages and to avoid the disadvantages.

\subsection{Comparing predicative locking with predicative validation}

The following discussion of predicative locking and predicative validation extends a discussion in [Brägger and Reimer, 1983]. The disadvantages of predicative locking compared to predicative validation are:

- If predicative locking grants locks which are larger than necessary, then parallel transactions are blocked although they do not conflict. If on the other hand predicative locking requires locks as small as possible, then the time for checking lock compatibility may become very long.

- Every lock compatibility check has to be performed on a global data structure and has to be synchronized against lock compatibility checks of parallel transactions. This decreases parallelism.

- Incremental locking may lead to deadlocks. On the other hand, a deadlock-free locking policy reduces parallelism.

\footnotetext{
'The read set forthermore contains the subrelations characterizing the modified parts of the database relation.
}

- Two-phnse locking requires to lock objects longer than they are accessed. This delays other transactions which conld run in parallel under predicative validation.

The advantages of predicative locking over predicative validation are

- If the transactions lock subrelations described by a simple selection condition, then it is faster to check whether these subrelations overlap or not than to use queries during the validation pliase.

- Validating transactions are backed up and restarted, if serializability is endangered. With an increasing number of conflicts, this leads to a considerable amount of unsuccessful transactions. These unsuccessful transactions withdraw resources like CPU-time nud disk nccesses from successful transactions.

Performance evaluations using an implementation of both strategies in one single system, i.e. the DBPL database system developed at the University of Frankfurt [Böttcher, 1989], confirm especinlly what follows: Predicative locking is superior to predicative validation for histories with a high ratio of conflicts, wherens predicative validation performs better when very few conflicts oceur (see also section 4.3). Therefore, it seems to be advantageous to integrate both synchronization strategies and to cloose the appropriate synchronization strategy depending on the expected amounts of conflicts. This appronch is described in the next subsection.

\subsection{Integrated synchronization based on predicative validation and predicative locking}

We first ontline the iden of the integrated syuch ronization and afterwards give an example.

A key to the idea behind the presented integration is that the conflict probability of operations depends on the size of the subrelations accessed by the operations, i.c. usually tuple operations have a lower conflict probability than set-oriented operations accessing large parts of a relation.

A transaction may contain some operations with a lower conflict probability, i.e. tuple operntions, and other operations with a higher conflict probnbility, e.g. set-oriented operations. Therefore, we do not require that this transaction uses the same synclironization strategy for ench of its operntions. Instead, we allow that some operations are synchronized by validation, whereas other operations of the same trausaction are 


\begin{tabular}{|l||l|l|}
\hline & tuple operation & set-oriented operation \\
\hline \hline tuple operation & (predicative) validation & (predicative) locking \\
\hline set-oriented operation & (predicative) locking & (predicative) locking \\
\hline
\end{tabular}

Fignre 1: Selected Synchronization strategy depending on the operations.

synchronized by locking. This means that the synchronization strategy needs not to be chosen at the level of transactions, instead it can be chosen at the level of
operations.

Furthermore, a given operation (e.g. a tuple operation) may have different conflict probabilities with different other operations, i.c. conflicts with other tuple operations are less probable than conflicts with set-oriented operations. Therefore, we allow that the given operation is synchronized with one strategy (say validation) against other tuple operations and with another strategy (say locking) against set-oriented operations.

The overall idea behind the integration is the following The scheduler determines the adequate synchronization strategy for each pair of possibly conflicting operations of different transactions. If the conflict probability of this pair of operations is high, then the operations are synchironized by (predicative) locking, if on the other hand the conflict probability of this pair of operations is low, then the operations are synchronized by (pred-

Since the scheduler has to decide for every pair of operit needs a ver their conflict probability is high or low heuristics which is implentistics for that decision. The system in order to select thed in the DBPL database works as follows. The heurie synchronization strategy of operations on database rics distinguishes two kinds database programming relations supported by the tions and set-oriented operange DBPL: tuple operntions are only in conflict, if thes. Two tuple operatuple. That is why the if they operate on the same probability of conflicts betweistics assumes, that the is low. On the other hetween two tuple operations conflicts with another ope, a set-oriented operation relations of both operationstion, if the accessed sub. heuristics assure operations overlap. That is why the tween two operation the probability of conflicts be a set-oriented operation.

The heris

nism: Each tuple be regarded as a voling mechaeach set-oriented operation votes for validation and more, the vote for locking votes for locking. Furtherdation. Hence, if both operations are tuple operations, both operations vole for validation and they are vali. dated against ench other. If however onle opcration is a set-oriented operation, then this operation votes for locking and both operations are synchronized against ench other by locking. The heuristics is summarized in the table given in figurel.

Now we give an example in order to illustrate the heuristics: Consider three transections T1, T2, T3 ac. cessing the same relation. T1 and T2 perform a tuple operation and T3 performs a set-oriented operation. Then the DBPL heuristics nssumes that the couflict probability between the operations of $\mathrm{T} 1$ and $\mathrm{T} 2$ is low, while the conflict probability of the operation of $\mathrm{T} 3$ with the operation of $\mathrm{T} 1$ (and the operation of $\mathrm{T}$ ? respectively) is high. This means, the DBPL schednles synchronizes the operations of $\mathrm{T} 1$ and $\mathrm{T} 2$ by vnlidation, whereas the set-oriented operation of $\mathrm{T} 3$ is synchronized against the tuple operation of $\mathrm{Tl}$ (and the tuple operation of $\mathrm{T} 2$ respectively) by (predicative) locking

In the integrated synchronization ${ }^{3}$, one of both strate gies (predicative locking or predicative validation) can be chosen for any single pair of operations independent from the synchronization strategies chosen for othet pairs of operations. The correctness of this integrated synchronization is stated in the following theorem.

Theorem:

If a scheduler uses the algorithm outlined in section 4.2 and synchronizes each pair of conflicting operations of different transactions either by (predicative) two-plinse locking or by (predicotive) validation, then every history produced by the scheduler is serializable.

The proof of this theorem is given in [Böttcher, 1989]. It is an extension of the serializnbility proof for two-phase locking, which can be found e.g. in [Bernstein et al., 1987]. Since the proof does not de pend on properties of predicntive synchronization, the theorem holds for physical synchronization as well.

'The implenentation of the scheduler based on inte grated synchronization is described in the sections 4.1 and 4.2. 


\begin{tabular}{|l||c|c|c|c|}
\hline & p-read-lock & p-write-lock & e-rend-lock & e-write-lock \\
\hline \hline p-read-lock & compatible & compatible & compatible & not compatible \\
\hline p-write-lock & compatible & compatible & not compatible & not compatible \\
\hline e-read-lock & compatible & not compatible & compatible & not compatible \\
\hline e-write-lock & not compatible & not compatible & not compntible & not compatible \\
\hline
\end{tabular}

Figure 2: Compatibility of p-locks and c-locks.

\section{Implementation and experimental results}

Having outlined the ider behind integrated predicative synchronization, we now describe the implementation of the heuristics within the DBPL database system, sketch the transaction scheduling nlgorithm, and present a performance test, which demonstrates that at least for the tested transaction lond the integrated scheduling is superior to both predicative locking and predicative validation.

\subsection{Implementation of the heuristics}

Since predicative validation is alrendy described in [Reimer, 1983] and the fast theorem prover used for lock compatibility checks in the DBPL database sys$\mathrm{tem}$ is described in [Böttcher et al, 1986], here we restrict the implementation description to the heuristics selecting the synchronization strategy.

In order to implement the heuristics, the lock trble of the DBPL database system distinguishes between two kinds of locks, exclusive locks (e-locks) and participation locks ( $p$-locks). e-locks forbid other locks on overlapping parts of a relation, whereas p-locks forbid e-locks on overlapping parts of a relation, but allow for other p-locks on the same relation. Pairs of operations which are locked by p-locks are synchronized by predicative validation. The heuristics uses e-locks for setoriented operations and p-locks for tuple operations. The lock compatibility is summarized in the table of figure 2 which also distinguishes read locks and write locks. This compatibility matrix guarantees that plocks only have to be checked against e-locks but not against other p-locks. Hence, if two operations are synchronized by validation, i.c. they use only p-locks, then no lock compatibility check ${ }^{4}$ is needed. Lock compatibility only has to be checked, if two operations have a high conflict probability and are synchronized by predicative locking, i.e. if at least one of them is a setoriented operation and requires an e-lock.

\footnotetext{
That is the most expensive operation of predicative locking.
}

In a similar way validation is restricted to be performed only for those pairs of operations, with a low conflict probability, i.e. tuple operntions for which n p-lock was required. In order to implement this restricted validation, the integrated scheduler keeps in the rend sets only used subrelations of the operations with a low confict probability, and similnrly it transfers only the old and new values of these operntions into the write sets. Since the integrated synchronization does not collect the old and new values of set-oriented write operations in the write sets of the Iransactions (as predicative valjdation does), queries during the validation phase are applied to mucli smaller write sets than this is the case with predicative validation.

To summarize: Every pair of conflicting operations is synchronized by only one synchronization strategy, predicative locking or predicative validation. $\Lambda$ fast heuristics selects the approprinte strategy depending on the expected conflict probnbility, i.e. depending on the kinds of both operations. If the conflict probability between them is ligh, then locking is applied. If on the other hand operations are syuchronized by valida tion using queries, then these queries only linve to be applied to small write sets.

\subsection{The scheduling algorithm for integrated synchronization}

In the following sketch of the transaction scheduling algorithm, $<$; denotes that $A$ is executed in a critical section. The algorithm is a combination of the parallel validation algorithm of |Kung and Robinson, 1981], the two-phase locking algorithm and the two-phase commit protocol [Bernstein et al, 1987]:

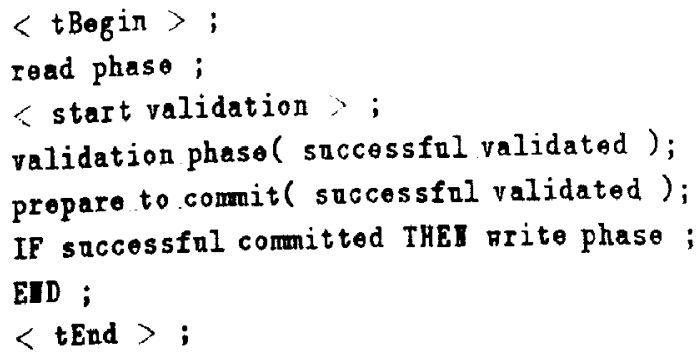


The procedures

succossful comitod proparo to commit and mit protocol |Bemstein element the two-phase com(B) procedures tBogin, start ralidation and tEnd are implemented ns described in [Kung and Robinson, 1981]. During the read phase write operations are performed on local copies. Read locks are required immediately before the read operations, and write locks are required at the end of the read phose (before start _validation). ${ }^{5}$ All locks nre released at the end of transaction (before tEnd). In the validation phase only tuple operations ( $\mathrm{p}$-locked oprations) are validated against other tuple operations (p-locked operations). Therefore, we expect that the validation time of integrated synchronization is rather short compared to ordinary validation.

\subsection{A performance test within the
DBPL system}

Performance results comparing predicative locking and predicative validation can not be expected to be the ienl validation, becaisen of physical locking and phys. ple lock compatibility physical locking uses a sinpredieative locking whecking mechanism whereas [Bötcher et al., 1986]) in order to cheorem prover (c.g. bility

A performance comparison of predicative locking and of both synchronization strategies validation uses CPU.time strategies. E.g., predicative nborted iransactions, time and database accesses for not use these resources for wedicative locking does thermore, predicative locking use transactions. Furfor checking lock compatibilis uses a theorem prover idntion uses query cunluation, while predicntive val. Hence it seems to be very ion the validation phase. which not only considers difficult to develop a model synchronization strategies but aferences between both them Modelling the cost for also correctly weights linn nay be even more for integrated synchronizapteferred to implement all three strated. Therefore, we single dniabnse systemb three strategies within one mance. This was done on a to compare their perforThe performance test is as follows. We number of processes (from tollows. We liave a varying ting the same kind of transactions infuitely submitperforms two write operations ons. Each transaction integrity check containing a complex tuples and an

'For the teason see the correct

'We ased the DBPL databiness proof in the appendix. Oniversity of Frantfart. (written in DBPJ, [Schmidt and Matthes, 1990]) is as follows.

\section{IOT SOHE $t 1$ II $R 1$ SOHE $t 2$ II $R 2$ SOHE $t 3$ II $R 3$ \\ ( $(t 1$.attI12 $=t 2 \cdot \operatorname{attr12})$ AID \\ (t1.attr13 $=$ t3.attr13) AID \\ (t2.attr23 $=t 3 . \operatorname{attr23})$}

Whenever locking is used, the subrelations locked for the write operations overlnp with the subrelations locked for the query. For the predicative vnlidation the test distinguishes two coses, first that no conflict occurs at all, and second the more realistic case that some conflicts occur. ${ }^{7}$

We count the number of successful completed transac. tions within a fixed time interval (transaction trouglput) and compare the integrated synchronization with predicative locking and predicative validation. The following diagram shows the transaction throughput depending on the number of parnllel processes which submit transactions.

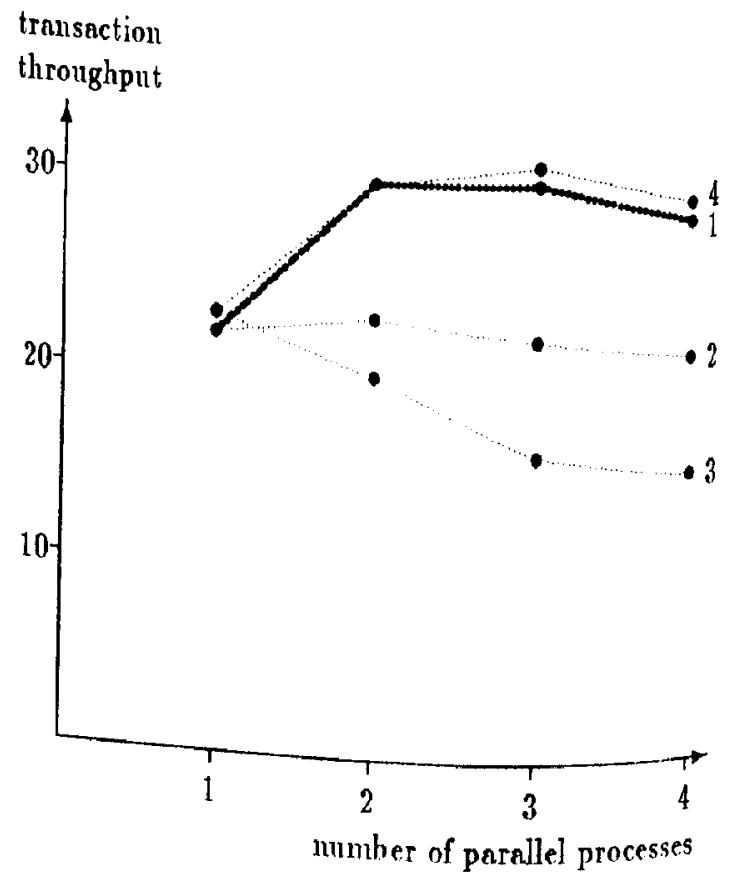

Line 1 denotes the integrated synchronization, line 2 denotes predicative locking,

line 3 denotes predicntive validntion with conflicts, line 4 denotes predicative vnlidation without conflicts.

In this performance test, predicative validation wins if no conflicts occur at all, but looses if conflicts be tween the query and the write operations of parallel ${ }^{7}$ This distin

scheduling, since does not apply to locking or integrated ways overlap with the subrelations locked for the query al operations. 
transactions occur. Integrated synchronization is superior to both predicative locking, and it is also superior to predicative validation, if there occur some conflicts. This performance evaluation has been confirmed by other performance evaluations within the DBPL systen which yield similar results [Kupijai, 1988].

\section{Summary and Conclusion}

We have compared predicative locking and predicative validntion and have shown, that predicative locking is superior to predicative validation if many conflicts occur, whereas predicative validation performs better if no conflicts occur. In order to combine the advantages of both synchronization methods and to avoid their disad vantages, we have developed the integrated synchronization. Integrated synchronization uses a fast heuristics and schedules pairs of operations depending on their conflict probability: Pairs of operations with a low conflict probability, i.e. pairs of tuple operntions, are validated against each other, whereas pairs of operations with $n$ high conflict probability, i.e. pairs including at least one set-oriented operation, are synchronized by predicative locking.

Furthermore, we have implemented all three synchronization strategies, predicative locking, predicative validation and the integrated synchronization within the federated DBPL database system and compared their performance. The presented result shows that integrated synchronization is superior to both predicative locking and predicative validation. Altogether, integrated synchronization seems to be an important improvement to schedulers of federnted database systems.

\section{References}

[Bernstein et al., 1981] P.A. Bernstein, N. Goodman, and M.Y. Lai. Laying Phantoms to Rest (By Understanding the Interactions Between Schedulers and Translators in a Database System). Technical Report, Harvard University, Aiken Computation Laboratory, Cambridge, 1981.

[Bernstein et al, 1987] P.A. Bernstein, V. Hadzilacos, and N. Goodman. Concurrency Control and Reconery in Database Systems. Addison-Wesley, 1987.

[Böttcher, 1989] S. Böttcher. Prädikative Selektion als Grandlage für Transaktionssynchronisation und Datenintegrität. PhD thesis, FB Informatik, Univ. Frankfurt, 1989.

[Böttcher, 1990a] S. Böttcher. Attribute inheritance implemented on top of a relational database sys- tem. In M. Liu, editor, Proc. $\sigma^{\text {th }}$ International Conference on Data Engincering, Los Angeles, Cnlifornin, USA, 1990.

[Böttcher, 1990h] S. Böttcher. Inproving the concurrency of integrity checks and write operations. In S. Abiteboul and P. Kanmellakis, editors, Proc. Third International Conference on Database Theory, Paris, France, 1990. (to nppear)

[Böttcher et al, 1986) S. Böttcher, M. Jarke, and J.W. Schmidt. Adaptive predicate managers in database systems. In Frocedings of the $12^{\text {th }}$ International Conferencr on Very Large Data Bases, Kyoto, Japan, 1986.

[Brägger and Reimer, 1983] R.P. Brägger nnd $M$. Reimer. Predicative Scheduling: Integration of Locking and Optimistic Methods. Report 53, ETH Züricli, 1983.

[Eswaran el al., 1976] K.P. Eswaran, J.N. Gray, R.A. Lorie, and I.L. Traiger. The notions of consistency and predicate locks in a database system. Com. munications of the $A C M, 19(11), 1976$.

[Franaszek and Robinson, 1985] P. Franaszek and J.T. Robinson. Limitations of concurrency in transaction processing. ACM ToDS, 10(1), 1985.

[Kung and Robinson, 1981] H.T. Kung and J.T. Robinson. On optimistic methods for concurrency control. ACM ToDS, 26(2), 1981.

[Kupijni, 1988] N. Kupijaj. Kombination und Mischung von prädikativem Sperren und prädikativer Validierung. Diplomn thesis, University of Frankfurt, 1988 .

[Papndinitrion, 1986] C.H. Papadimitrion. The Theory of Dutabase Concurrency Control. Comprter Science Press, Rockville, 1980.

[Reimer, 1983] M. Reimer. Solving the phantom problem by predicative optinustic concurrency control. In Proceedings of the $g^{\text {th }}$ International Conference on Very Large Data Bases, Firence, Itnly, 1983.

[Schnidt and Matthes, 1990] J.W. Sclumidt and F. Mattlies. DBPL Language and System Manual. In. Document, Univ. Hamburg, 1990. 